Georgian Mathematical Journal

Volume 8 (2001), Number 2, 347-362

\title{
HÖLDER VERSIONS OF BANACH SPACE VALUED RANDOM FIELDS
}

\author{
ALFREDAS RAČKAUSKAS AND CHARLES SUQUET
}

\begin{abstract}
For rather general moduli of smoothness $\rho$, like $\rho(h)=h^{\alpha} \ln ^{\beta}(c / h)$, we consider the Hölder spaces $H_{\rho}(B)$ of functions $[0,1]^{d} \rightarrow B$ where $B$ is a separable Banach space. We establish an isomorphism between $H_{\rho}(B)$ and some sequence Banach space. With this analytical tool, we follow a very natural way to study, in terms of second differences, the existence of a version in $H_{\rho}(B)$ for a given random field.
\end{abstract}

2000 Mathematics Subject Classification: 60G05, 60G17, 60G15.

Key words and phrases: Banach valued Brownian motion, central limit theorem, Schauder decomposition, second difference, skew pyramidal basis.

\section{INTRODUCTION}

Let $\rho(\delta), 0 \leq \delta \leq 1$ be a modulus of smoothness and $B$ a separable Banach space. Denote by $H_{\rho}(B)=H_{\rho}\left([0,1]^{d} ; B\right)$ the space of all functions $x:[0,1]^{d} \rightarrow$ $B$ such that

$$
\|x(t+h)-x(t)\|_{B}=O(\rho(|h|))
$$

uniformly in $t \in[0,1)^{d}$. $H_{\rho}^{o}(B)=H_{\rho}^{o}\left([0,1]^{d} ; B\right)$ is the subspace of functions for which $O(\rho(|h|))$ can be replaced by $o(\rho(|h|))$ in (1.1). Equipped with the related Hölder norm (precise definitions are given in Section 2), $H_{\rho}(B)$ becomes a non-separable Banach space with $H_{\rho}^{o}(B)$ as a closed separable subspace. Letting the modulus $\rho$ vary gives a very natural scale of spaces allowing us to classify by their global regularity the functions more than continuous. This functional framework is interesting in the theory of stochastic processes since very often the continuous stochastic process under study has a better regularity than the bare continuity. Moreover, for obvious topological reasons, weak convergence in Hölder spaces is stronger than in the classical space $C\left([0,1]^{d} ; B\right)$ of $B$ valued continuous functions on $[0,1]^{d}$.

In the previous papers [11], [10], the authors discussed the following problem in the special case $B=\mathbb{R}$ :

(I) For a given real valued random field indexed by $[0,1]^{d}$, find sufficient conditions for the existence of a version with sample paths in $H_{\rho}^{o}(B)$.

The present contribution studies (I) in the general case of a separable Banach space $B$. The history of this problem began with the well known Kolmogorov's 
condition: if the real valued stochastic process $(\xi(t), t \in[0,1])$ satisfies

$$
P(|\xi(t+h)-\xi(t)|>\lambda) \leq c \lambda^{-\gamma} h^{1+\varepsilon},
$$

where $c, \varepsilon>0$ and $\gamma>1$ are constants, then it has a version with almost surely continuous paths. This was the root of successive generalizations leading to processes indexed by an abstract parameter set $T$. The modern theory expresses condition for the existence of a continuous version in terms of the geometry of $T$, i.e., some metric entropy or majorizing measure condition which estimates the size of $T$ with respect to a pseudo metric related to $\xi$. The study of Hölder regularity is another branch stemming from Kolmogorov's condition. Indeed, (1.2) is sufficient for $\xi$ to have a version with sample paths in the Hölder space $H_{\rho}^{o}([0,1] ; \mathbb{R})$ with $\rho(h)=|h|^{\alpha}$ for any $0<\alpha<\varepsilon / \gamma$. Ciesielski [3] gave sufficient conditions for a Gaussian process to have a version with $\alpha$-Hölderian paths. Using the method of triangular functions, Delporte [4] established sufficient conditions for the existence of a version of $\xi$ in $H_{\rho}^{o}([0,1] ; \mathbb{R})$ for general moduli of smoothness $\rho$. Ibragimov [6] and Nobelis [9] studied the problem (I) for general $\rho$ and $B=\mathbb{R}$.

The method of triangular functions used by Ciesielski [2, 3], Delporte [4], Kerkyacharian and Roynette [7] relies on the following well known decomposition of a real valued continuous function $x$ :

$$
x(t)=\sum_{j=0}^{\infty} \sum_{v \in V_{j}} \lambda_{j, v}(x) \Lambda_{j, v}(t), \quad t \in T,
$$

where $\Lambda_{j, v}$ 's are the Faber-Schauder triangular functions and $V_{j}$ is the set of dyadic numbers of level $j$ in $T=[0,1]$. In fact the triangular functions form a basis (in Schauder's sense) of $H_{\rho}^{o}([0,1] ; \mathbb{R})$ when $\rho(h)=|h|^{\alpha}$. Moreover, the Hölder regularity of a continuous function is characterized by the rate of decreasing of its coefficients $\lambda_{j, v}(x)$ in this basis. This provides the Ciesielski [2] isomorphisms between these Hölder spaces and some sequence spaces. These isomorphisms give a very convenient discretization procedure in [10] to study (I). In [11] we use the same method, replacing the basis of triangular functions by the basis of skew pyramidal functions defined on $T=[0,1]^{d}$, denoted again by $\Lambda_{j, v}$ and indexed by the dyadic points $v$ of level $j$ in $[0,1]^{d}$. The scalar coefficients $\lambda_{j, v}(x)$ are some dyadic second differences of $x$. In the present contribution, we keep formally the same decomposition (1.3) of $B$-valued Hölder functions into series of pyramidal functions. But now the pyramidal functions are scalar while their coefficients are vectors lying in $B$. The important fact is the preservation in this new setting of the equivalence between the initial Hölder norm $\|x\|_{\rho}$ with the sequential norm

$$
\|x\|_{\rho}^{\mathrm{seq}}:=\sup _{j \geq 0} \frac{1}{\rho\left(2^{-j}\right)} \max _{v \in V_{j}}\left\|\lambda_{j, v}(x)\right\|_{B} .
$$

Of course, this cannot be the question of any Schauder basis for $H_{\rho}^{o}(B)$ in the general case since $B$ itself does not necessarily possess such a basis. 
From (1.3) and (1.4) it should be clear that the control of the coefficients $\lambda_{j, v}(x)$ is the key tool in our study. It is then relevant to state the basic asumptions in problem (I) in terms of the second differences

$$
\Delta_{h}^{2} \xi(t):=\xi(t+h)+\xi(t-h)-2 \xi(t)
$$

of the random fields considered. This brings more flexibility than the classical use of first increments (for more argumentation see [10]).

The paper is organized as follows. Section 2 exposes the analytical preliminaries: expansion in a series of pyramidal functions, equivalence of norms. In Section 3, we discuss problem (I) of the existence of Hölderian versions. The systematic use of the sequential norm (1.4) enables us to provide sufficient conditions whose general form may be sketched as follows. Assuming some uniform control of second differences like

$$
P\left(\left\|\Delta_{h}^{2} \xi(t)\right\|_{B}>r \sigma(|h|)\right) \leq \Psi(r), \quad r>0,
$$

we consider the series

$$
R(u):=\sum_{j=0}^{\infty} 2^{j d} \Psi\left(u \frac{\rho}{\sigma}\left(2^{-j}\right)\right) .
$$

Then the convergence of $R\left(u_{0}\right)$ for some $u_{0}>0$ gives the existence of a version of $\xi$ in $H_{\rho}(B)$, while the convergence for every $u>0$ gives a version in $H_{\rho}^{o}(B)$. When (1.6) is obtained through weak $p$-moments, the corresponding result improves the Ibragimov's classical result for the case $B=\mathbb{R}$ by a logarithmic factor in the modulus $\rho[6]$. When (1.6) is verified through exponential Orlicz norms, we have practical conditions precise enough to discriminate between $H_{\rho}(B)$ and $H_{\rho}^{o}(B)$. For instance, our conditions detect the optimal Hölder regularity of $B$-valued Brownian motions. Examples of Ornstein-Uhlenbeck processes in $c_{0}$ and of $p$-stable processes in $\ell^{r}$ illustrate our general results.

\section{Analytical Background}

Throughout $T=[0,1]^{d}$ and $\mathbb{R}^{d}$ is endowed with the norm $|t|:=\max _{1 \leq i \leq d}\left|t_{i}\right|$, $t=\left(t_{1}, \ldots, t_{d}\right) \in \mathbb{R}^{d}$. Denote by $B$ a Banach space with the norm $\|\cdot\|_{B}$ and by $H_{\rho}(B)=H_{\rho}(T ; B)$ the set of $B$-valued continuous functions $x: T \rightarrow B$ such that $\omega_{\rho}(x, 1)<\infty$, where

$$
\omega_{\rho}(x, \delta):=\sup _{t, s \in T, 0<|t-s|<\delta} \frac{\|x(t)-x(s)\|_{B}}{\rho(|s-t|)}
$$

and $\rho$ is a modulus of smoothness satisfying conditions (2.1) to (2.5) below.

The technical conditions required for $\rho$ are the following, where $c_{1}, c_{2}$ and $c_{3}$ are positive constants:

$$
\begin{aligned}
& \rho(0)=0, \rho(\delta)>0,0<\delta \leq 1 \\
& \rho \text { is non-decreasing on }[0,1] \\
& \rho(2 \delta) \leq c_{1} \rho(\delta), \quad 0 \leq \delta \leq 1 / 2
\end{aligned}
$$




$$
\begin{aligned}
& \int_{0}^{\delta} \frac{\rho(u)}{u} d u \leq c_{2} \rho(\delta), \quad 0<\delta \leq 1 \\
& \delta \int_{\delta}^{1} \frac{\rho(u)}{u^{2}} d u \leq c_{3} \rho(\delta), \quad 0<\delta \leq 1 .
\end{aligned}
$$

To show a concrete class of functions satisfying the conditions $(2.1)-(2.5)$, let us define inductively the sequence of functions $\ell_{k}$ on $\left(u_{k}, \infty\right)$ by $\ell_{1}(u)=\ln u$, $u_{1}=1$ and, for $k \geq 2, \ell_{k}(u)=\ln \left(\ell_{k-1}(u)\right)$, and $u_{k}$ such that $\ell_{k-1}\left(u_{k}\right)=1$.

Elementary computations show that for any $\alpha \in(0,1)$ and any finite sequence $\beta_{1}, \ldots, \beta_{m} \in \mathbb{R}$ there exists a set of positive constants $b_{1}, \ldots, b_{m}$ such that the functions

$$
\rho(h)=h^{\alpha} \prod_{k=1}^{m} \ell_{k}^{\beta_{k}}\left(b_{k} / h\right), \quad 0<h \leq 1,
$$

satisfy conditions (2.1) to (2.5).

The set $H_{\rho}(B)$ is a Banach space when endowed with the norm

$$
\|x\|_{\rho}:=\|x(0)\|_{B}+\omega_{\rho}(x, 1) .
$$

Obviously, an equivalent norm is obtained by replacing $\|x(0)\|_{B}$ in the above formula by $\|x\|_{\infty}:=\sup \left\{\|x(t)\|_{B} ; t \in T\right\}$. Define

$$
H_{\rho}^{o}(B)=H_{\rho}^{o}(T ; B):=\left\{x \in H_{\rho}(B): \lim _{\delta \rightarrow 0} \omega_{\rho}(x, \delta)=0\right\} .
$$

Then $H_{\rho}^{o}(B)$ is a closed subspace of $H_{\rho}(B)$. Now let us remark that for any function $\rho$ satisfying (2.1) and (2.5) there is a positive constant $c_{4}$ such that

$$
\rho(\delta) \geq c_{4} \delta, \quad 0 \leq \delta \leq 1
$$

Hence the spaces $H_{\rho}^{o}(B)$ always contain all the Lipschitz $B$-valued functions and in particular the (continuous) piecewise affine functions. When $B$ is itself separable, the separability of the spaces $H_{\rho}^{o}(B)$ follows by interpolation arguments.

Since we are interested in the analysis of these spaces in terms of second differences of the functions $x$, our first task is to establish the equivalence of the norm $\|x\|_{\rho}$ with some sequential norm involving the dyadic second differences of $x$. Our main reference for this part is Semadeni [14].

The so-called skew pyramidal basis was introduced by Bonic, Frampton and Tromba [1] and, independently, by Ciesielski and Geba (see the historical notes in $[14$, p. 72]). The reader is referred also to our previous contribution [11] for a more detailed explanation.

If $A$ is a convex subset of $T$, the function $f: T \rightarrow B$ is said to be affine on $A$ if it preserves the barycenter, i.e., for any finite sequence $u_{1}, \ldots, u_{m}$ in $A$ and nonnegative scalars $r_{1}, \ldots, r_{m}$ such that $\sum_{i=1}^{m} r_{i}=1, f\left(\sum_{i=1}^{m} r_{i} u_{i}\right)=\sum_{i=1}^{m} r_{i} f\left(u_{i}\right)$.

To explain the construction of the skew pyramidal basis, define first the standard triangulation of the unit cube $T=[0,1]^{d}$. Write $\Pi_{d}$ for the set of permutations of the indexes $1, \ldots, d$. For any $\pi=\left(i_{1}, \ldots, i_{d}\right) \in \Pi_{d}$, let $\Delta_{\pi}(T)$ be the 
convex hull of $d+1$ points $0, e_{i_{1}},\left(e_{i_{1}}+e_{i_{2}}\right), \ldots, \sum_{k=1}^{d} e_{i_{k}}$, where $e_{i}$ 's are the vectors of the canonical basis of $\mathbb{R}^{d}$. So, each simplex $\Delta_{\pi}(T)$ corresponds to one path from 0 to $(1, \ldots, 1)$ via the vertices of $T$ and such that along each segment of the path, only one coordinate increases while the others remain constant. Thus $T$ is divided into $d$ ! simplexes with disjoint interiors. The standard triangulation of $T$ is the family $T_{0}$ of simplexes $\left\{\Delta_{\pi}(T), \pi \in \Pi_{d}\right\}$.

Next, we divide $T$ into $2^{j d}$ dyadic cubes with edge $2^{-j}$. By dyadic translations and change of scale, each of them is equipped with a triangulation similar to $T_{0}$. And $T_{j}$ is the set of $2^{j d} d$ ! simplexes so constructed.

For $j \geq 1$ the set $W_{j}:=\operatorname{vert}\left(T_{j}\right)$ of vertices of the simplexes in $T_{j}$ is

$$
W_{j}=\left\{k 2^{-j} ; 0 \leq k \leq 2^{j}\right\}^{d} .
$$

In what follows we put $V_{0}:=W_{0}$ and $V_{j}:=W_{j} \backslash W_{j-1}$. So $V_{j}$ is the set of new vertices born with the triangulation $T_{j}$. More explicitly, $V_{j}$ is the set of dyadic points $v=\left(k_{1} 2^{-j}, \ldots, k_{d} 2^{-j}\right)$ in $W_{j}$ with at least one $k_{i}$ odd.

The $T_{j}$-pyramidal function $\Lambda_{j, v}$ with peak vertex $v \in V_{j}$ is the real valued function defined on $T$ by three conditions:

i) $\Lambda_{j, v}(v)=1$;

ii) $\Lambda_{j, v}(w)=0$ if $w \in \operatorname{vert}\left(T_{j}\right)$ and $w \neq v$;

iii) $\Lambda_{j, v}$ is affine on each simplex $\Delta$ in $T_{j}$.

Observe that the notation $\Lambda_{j, v}$ is somewhat redundant and could be simplified in $\Lambda_{v}$ since $V_{j}$ 's form a partition of the set of dyadic points of $[0,1]^{d}$.

It follows clearly from the above definition that the support of $\Lambda_{j, v}$ is the union of all simplexes in $T_{j}$ containing the peak vertex $v$. By [14, Prop. 3.4.5], the functions $\Lambda_{j, v}$ are obtained by dyadic translations and changes of scale:

$$
\Lambda_{j, v}(t)=\Lambda\left(2^{j}(t-v)\right), \quad t \in T, v \in V_{j},
$$

from the same function $\Lambda$ with support included in $[-1,1]^{d}$ :

$$
\Lambda(t):=\max \left(0,1-\max _{t_{i}<0}\left|t_{i}\right|-\max _{t_{i}>0} t_{i}\right), \quad t=\left(t_{1}, \ldots, t_{d}\right) \in \mathbb{R}^{d} .
$$

The $B$-valued coefficients $\lambda_{j, v}(x)$ are given by

$$
\begin{aligned}
& \lambda_{0, v}(x)=x(v), \quad v \in V_{0} ; \\
& \lambda_{j, v}(x)=x(v)-\frac{1}{2}\left(x\left(v^{-}\right)+x\left(v^{+}\right)\right), \quad v \in V_{j}, j \geq 1,
\end{aligned}
$$

where $v^{-}$and $v^{+}$are defined as follows. Each $v \in V_{j}$ admits a unique representation $v=\left(v_{1}, \ldots, v_{d}\right)$ with $v_{i}=k_{i} / 2^{j},(1 \leq i \leq d)$. The points $v^{-}=\left(v_{1}^{-}, \ldots, v_{d}^{-}\right)$ and $v^{+}=\left(v_{1}^{+}, \ldots, v_{d}^{+}\right)$are defined by

$$
v_{i}^{-}=\left\{\begin{array}{ll}
v_{i}-2^{-j} & \text { if } k_{i} \text { is odd } \\
v_{i} & \text { if } k_{i} \text { is even; }
\end{array} \quad v_{i}^{+}= \begin{cases}v_{i}+2^{-j}, & \text { if } k_{i} \text { is odd } \\
v_{i} & \text { if } k_{i} \text { is even }\end{cases}\right.
$$


Since $v$ is in $V_{j}$, at least one of $k_{i}$ 's is odd, so $v^{-}, v$ and $v^{+}$are really three distinct points of $T$. Moreover, we can write

$$
v^{-}=v-2^{-j} e(v), \quad v^{+}=v+2^{-j} e(v) \quad \text { with } \quad e(v):=\sum_{k_{i} \text { odd }} e_{i},
$$

so $\lambda_{j, v}(x)$ is a second difference directed by the vector $e(v)$. Observe further that if $v$ is in $V_{j}$, then $v^{-}$and $v^{+}$are in $W_{j-1}$.

Note here that the sequences $\left(\lambda_{j, v}\right)$ and $\left(\Lambda_{j, v}\right)$ are biorthogonal in the following sense.

Lemma 1. For $j, j^{\prime} \geq 0$ and $v \in V_{j}, v^{\prime} \in V_{j^{\prime}}$,

$$
\lambda_{j, v}\left(\Lambda_{j^{\prime}, v^{\prime}}\right)=\delta_{v, v^{\prime}},
$$

where $\delta_{v, v^{\prime}}=0$ if $v \neq v^{\prime}, \delta_{v, v^{\prime}}=1$ if $v=v^{\prime}$ (and then $j=j^{\prime}$ ).

Proof. Suppose first $j$ positive, so

$$
\lambda_{j, v}\left(\Lambda_{j^{\prime}, v^{\prime}}\right)=\Lambda_{j^{\prime}, v^{\prime}}(v)-\frac{1}{2}\left(\Lambda_{j^{\prime}, v^{\prime}}\left(v^{-}\right)+\Lambda_{j^{\prime}, v^{\prime}}\left(v^{+}\right)\right),
$$

with $v \in V_{j}$ and $v^{+}, v^{-} \in W_{j-1}$.

Case $1: j<j^{\prime}$. Then $v, v^{+}$and $v^{-}$are the vertices of the triangulation $T_{j}$ and hence also of $T_{j^{\prime}}$, but none of them can be equal to $v^{\prime}$, so $\Lambda_{j^{\prime}, v^{\prime}}$ vanishes at $v, v^{+}, v^{-}$.

Case $2: j>j^{\prime}$. Then the segment $\left[v^{-}, v^{+}\right]$( with middle-point $v$ ) is contained in some simplex $\Delta$ of $T_{j^{\prime}}$ and $\Lambda_{j^{\prime}, v^{\prime}}$ is affine on $\Delta$, so $\lambda_{j, v}\left(\Lambda_{j^{\prime}, v^{\prime}}\right)=0$.

Case $3: j=j^{\prime}$. Then $v^{-}$and $v^{+}$are in $W_{j^{\prime}-1}$, so $\Lambda_{j^{\prime}, v^{\prime}}$ vanishes at $v^{-}$and $v^{+}$and $\lambda_{j, v}\left(\Lambda_{j^{\prime}, v^{\prime}}\right)=\Lambda_{j^{\prime}, v^{\prime}}(v)=\delta_{v, v^{\prime}}$, by i) and ii) in the definition of pyramidal functions.

To complete the proof, note that in the special case $j=0, \lambda_{0, v}\left(\Lambda_{j^{\prime}, v^{\prime}}\right)=$ $\Lambda_{j^{\prime}, v^{\prime}}(v)=\delta_{v, v^{\prime}}$, since $v$ is a vertex of $T_{0}$ and hence of $T_{j^{\prime}}$.

As usual, the space $C(T ; B)$ of continuous functions $x: T \rightarrow B$ is endowed with the uniform norm $\|x\|_{\infty}=\sup _{t \in T}\|x(t)\|_{B}$. Define the operators $E_{j}(j \geq 0)$ on the space $C(T ; B)$ by

$$
E_{j} x:=\sum_{i=0}^{j} \sum_{v \in V_{i}} \lambda_{i, v}(x) \Lambda_{i, v}, \quad x \in C(T ; B) .
$$

Lemma 2. The B-valued function $E_{j} x$ is affine on each simplex of $T_{j}$ and such that $E_{j} x(w)=x(w)$ for each $w \in W_{j}$.

Proof. Since each simplex $\Delta$ of $T_{j}$ is included in one simplex of $T_{i}$ for $i \leq j$, the first claim follows clearly from the fact that $\Lambda_{i, v}$ 's are affine on the simplexes of $T_{i}$.

We check the second claim by induction on $j$. First, for $w \in W_{0}$, we have by the definition of $\lambda_{0, v}$ 's and i), ii) of the definition of the pyramidal functions,

$$
E_{0} x(w)=\sum_{v \in V_{0}} x(v) \Lambda_{0, v}(w)=x(w) .
$$


Next assuming the interpolation property true for $j$, consider, for $w$ in $W_{j+1}$, the decomposition

$$
E_{j+1} x(w)=E_{j}(w)+\sum_{v \in V_{j+1}} \lambda_{j+1, v}(x) \Lambda_{j+1, v}(w) .
$$

If $w$ already belongs to $W_{j}$, then $E_{j} x(w)=x(w)$ by the induction hypothesis and the second term in the above decomposition vanishes by ii) since $w$ belonging to vert $\left(T_{j+1}\right) \backslash V_{j+1}$ cannot be a peak vertex for one of $\Lambda_{j+1, v}$. To complete the proof, it remains to treat the case $w \in V_{j+1}$. Using again i) and ii) we have

$$
\sum_{v \in V_{j+1}} \lambda_{j+1, v}(x) \Lambda_{j+1, v}(w)=\lambda_{j+1, w}(x)=x(w)-\frac{1}{2}\left(x\left(w^{-}\right)+x\left(w^{+}\right)\right) .
$$

Recall that $w=\frac{1}{2}\left(w^{+}+w^{-}\right)$. By [14, Lemma 3.4.8] there is at least one simplex $\Delta$ in $T_{j}$ having $w^{+}$and $w^{-}$as vertices. Since $E_{j} x$ is affine on $\Delta$ and $w^{-}$and $w^{+}$are in $W_{j}$, we have

$$
E_{j} x(w)=\frac{1}{2}\left(E_{j} x\left(w^{-}\right)+E_{j} x\left(w^{+}\right)\right)=\frac{1}{2}\left(x\left(w^{-}\right)+x\left(w^{+}\right)\right),
$$

and so $E_{j+1} x(w)=x(w)$.

Since the pyramidal functions are not $B$-valued, they cannot form a Schauder basis of $C(T ; B)$ as in the real valued case. Nevertheless we keep the same type of decomposition.

Proposition 1. Each $x$ in $C(T ; B)$ admits the series expansion

$$
x(t)=\sum_{j=0}^{\infty} \sum_{v \in V_{j}} \lambda_{j, v}(x) \Lambda_{j, v}(t), \quad t \in T,
$$

where the convergence holds in the strong topology of $B$ and is uniform with respect to $t$ on $T$.

Proof. For fixed $t$ there is a simplex $\Delta$ in $T_{j}$ containing $t$. Let $v_{0}, v_{1}, \ldots, v_{d}$ be the vertices of $\Delta$. Writing $\omega_{1}(\delta)$ for the modulus of continuity and recalling that the diameter of $\Delta$ is $2^{-j}$, we have

$$
\left\|x(t)-E_{j} x(t)\right\|_{B} \leq \omega_{1}\left(2^{-j}\right)+\left\|x\left(v_{0}\right)-E_{j} x(t)\right\|_{B} .
$$

Using the barycentric representation $t=\sum_{i=0}^{d} r_{i} v_{i}$ and Lemma 2, we get

$$
\left\|x\left(v_{0}\right)-E_{j} x(t)\right\|_{B}=\left\|\sum_{i=0}^{d} r_{i}\left(x\left(v_{0}\right)-x\left(v_{i}\right)\right)\right\|_{B} \leq \sum_{i=0}^{d} r_{i} \omega_{1}\left(2^{-j}\right)=\omega_{1}\left(2^{-j}\right) .
$$

The conclusion follows.

Now we are in the position to state the equivalence of norms we were looking for. For any function $x:[0,1]^{d} \rightarrow B$ the (possibly infinite) sequential seminorm is defined by

$$
\|x\|_{\rho}^{\text {seq }}:=\sup _{j \geq 0} \frac{1}{\rho\left(2^{-j}\right)} \max _{v \in V_{j}}\left\|\lambda_{j, v}(x)\right\|_{B} .
$$


Observe moreover that when $x$ is continuous, $\|x\|_{\rho}^{\text {seq }}=0$ if and only if $x=0$. In this case, $\|x\|_{\rho}^{\text {seq }}$ is a (possibly infinite) true norm.

Proposition 2. Under conditions (2.1) to (2.5), the norm $\|x\|_{\rho}$ is equivalent on $C(T ; B)$ to the sequential norm, i.e., there are positive constants $a, b$ such that for every $x \in C(T ; B)$,

$$
a\|x\|_{\rho}^{\mathrm{seq}} \leq\|x\|_{\rho} \leq b\|x\|_{\rho}^{\mathrm{seq}},
$$

with finite values of the norms if and only if $x \in H_{\rho}(B)$.

Proof. The proof is exactly the same as for [11, Prop. 1], replacing $|x(t)|$ by $\|x(t)\|_{B}$. Note that in this proof the continuity of $x$ was used through its expansion in a series of pyramidal functions which is now replaced by Proposition 1. Conditions (2.4) and (2.5) are not required for the inequality $a\|x\|_{\rho}^{\text {seq }} \leq\|x\|_{\rho}$ which holds true with $a=\min (1, \rho(1))$ for the most general moduli of smoothness $\rho$.

Remark. It is worth noticing that

$$
\left\|x-E_{J} x\right\|_{\rho}^{\mathrm{seq}}:=\sup _{j>J} \frac{1}{\rho\left(2^{-j}\right)} \max _{v \in V_{j}}\left\|\lambda_{j, v}(x)\right\|_{B}
$$

is non-increasing in $J$.

The next result is the key tool for the problem of existence of Hölderian versions for a given $B$-valued random field.

Proposition 3. Let $\theta=\left(\theta_{j, v} ; j \geq 0, v \in V_{j}\right)$ be a $B$-valued tree and consider the following conditions.

(a) $\sum_{j=0}^{\infty} \max _{v \in V_{j}}\left\|\theta_{j, v}\right\|_{B}<\infty$.

(b) $\sup _{j \geq 0} \frac{1}{\rho\left(2^{-j}\right)} \max _{v \in V_{j}}\left\|\theta_{j, v}\right\|_{B}<\infty$.

(c) $\lim _{J \rightarrow \infty} \sup _{j>J} \frac{1}{\rho\left(2^{-j}\right)} \max _{v \in V_{j}}\left\|\theta_{j, v}\right\|_{B}=0$.

Define the sequence $\left(S_{J}\right)_{J \geq 0}$ of continuous piecewise affine functions by

$$
S_{J}:=\sum_{j=0}^{J} \sum_{v \in V_{j}} \theta_{j, v} \Lambda_{j, v}
$$

Then under (a), $S_{J}$ converges in $C(T ; B)$ to some function $S$. Under (b), the same convergence holds and $S$ belongs to $H_{\rho}(T ; B)$. Under (c), $S_{J}$ converges in $H_{\rho}^{o}(T ; B)$.

Proof. The pyramidal functions being non-negative, we have

$$
\left\|\sum_{v \in V_{j}} \theta_{j, v} \Lambda_{j, v}(t)\right\|_{B} \leq \max _{v \in V_{j}}\left\|\theta_{j, v}\right\|_{B} \sum_{v \in V_{j}} \Lambda_{j, v}(t)
$$


for every $t \in T$. Recalling that $0 \leq \sum_{v \in V_{j}} \Lambda_{j, v}(t) \leq 1$ (cf., e.g., Lemma 2 in [11]), we see that (a) entails the uniform convergence on $T$ of $S_{J}$.

Under (b), the same estimate gives the convergence in $C(T ; B)$ of $S_{J}$ to a function $S$, provided that $\sum_{j \geq 0} \rho\left(2^{-j}\right)<\infty$. But this follows from our general assumption (2.4), comparing series with integral. Observe now that by Lemma $1, \lambda_{j, v}\left(S_{J}\right)=\theta_{j, v}$ if $j \leq J$, so by the continuity on $C(T ; B)$ of $\lambda_{j, v}$ 's, $\lambda_{j, v}(S)=\theta_{j, v}$ for every $j, v$. Applying now Proposition 2 to the continuous function $S$, we obtain $\|S\|_{\rho}<\infty$.

Suppose finally that $\theta$ satisfies (c) which is obviously stronger than (b). Then $S_{J}$ converges (at least) in $C(T ; B)$ sense to $S \in H_{\rho}(B)$ and $\lambda_{j, v}(S)=\theta_{j, v}$. Thus condition (c) means that $\left\|S-S_{J}\right\|_{\rho}^{\text {seq }}$ goes to zero, which gives the convergence of $S_{J}$ to $S$ in the $H_{\rho}(B)$ sense by Proposition 2. Moreover, $S_{J}$ 's are in $H_{\rho}^{o}(B)$ which is a closed subspace of $H_{\rho}(B)$, and so $S \in H_{\rho}^{o}(B)$.

The usefulness of Proposition 3 for the existence of Hölderian versions comes from its following obvious corollary.

Corollary 1. Let $x$ be any function $T \rightarrow B$ and define $\theta=\left(\lambda_{j, v}(x) ; j \geq\right.$ $\left.0, v \in V_{j}\right)$. Then $x$ coincides at the dyadic points of $[0,1]^{d}$ with a function $\bar{S}$ which is in $C(T ; B)$ under $(\mathrm{a})$, in $H_{\rho}(B)$ under $(\mathrm{b})$ and in $H_{\rho}^{o}(B)$ under $(\mathrm{c})$.

\section{Banach Valued Random Fields with Hölderian Versions}

We now consider a given $B$-valued random field $\xi=(\xi(t), t \in T)$, continuous in probability, and discuss the problem of existence of a version of $\xi$ with almost all paths in $H_{\rho}^{o}(B)$. In our setting, a natural candidate for such a version is of the form $\tilde{\xi}:=\sum_{j} \sum_{v \in V_{j}} \lambda_{j, v}(\xi) \Lambda_{j, v}$. Indeed, combining Corollary 1 with continuity in probability reduces the problem to the control of maxima of random coefficients $\left\|\lambda_{j, v}(\xi)\right\|_{B}$ which are norms of dyadic second differences of $\xi$. We define here the second difference $\Delta_{h}^{2} \xi(t)$ in a symmetrical form by

$$
\Delta_{h}^{2} \xi(t):=\xi(t+h)+\xi(t-h)-2 \xi(t), \quad t \in T, h \in C_{t},
$$

where

$$
C_{t}:=\left\{h=\left(h_{1}, \ldots, h_{d}\right) ; 0 \leq h_{i} \leq \min \left(t_{i}, 1-t_{i}\right), 1 \leq i \leq d\right\} .
$$

Theorem 1. Let $\xi=\{\xi(t), t \in T\}$ be a $B$-valued random field, continuous in probability. Assume there exist a function $\sigma:[0,1] \rightarrow \mathbb{R}^{+}, \sigma(0)=0$ and $a$ function $\Psi:(0, \infty] \rightarrow \mathbb{R}^{+}, \Psi(\infty)=0$ such that for all $r>0, t \in T, h \in C_{t}$,

$$
P\left(\left\|\Delta_{h}^{2} \xi(t)\right\|_{B}>r \sigma(|h|)\right) \leq \Psi(r) .
$$

Put for $0<u<\infty$,

$$
R(u)=R(\Psi, \sigma, \rho, u):=\sum_{j=0}^{\infty} 2^{j d} \Psi\left(u \frac{\rho}{\sigma}\left(2^{-j}\right)\right) .
$$

If $R\left(u_{0}\right)$ is finite for some $0<u_{0}<\infty$, then $\xi$ has a version in $H_{\rho}(B)$. If $R(u)$ is finite for every $0<u<\infty$, then $\xi$ has a version in $H_{\rho}^{o}(B)$. 
When $\Psi$ is non-increasing and $\sigma$ non-decreasing, the same conclusions hold if $R(u)$ is replaced by

$$
I(u):=\int_{0}^{1} \Psi\left(u \frac{\rho}{\sigma}(s)\right) \frac{d s}{s^{d+1}} .
$$

Proof. By Corollary 1 and continuity in probability, $\xi$ has a version in $H_{\rho}(B)$ if (and only if) $\|\xi\|_{\rho}^{\text {seq }}$ is finite almost surely, which is equivalent to

$$
\lim _{M \rightarrow \infty} P\left(\|\xi\|_{\rho}^{\text {seq }}>M\right)=0 .
$$

Using the obvious bound

$$
P\left(\|\xi\|_{\rho}^{\mathrm{seq}}>M\right) \leq \sum_{j=0}^{\infty} \sum_{v \in V_{j}} P\left(\left\|\lambda_{j, v}(\xi)\right\|_{B}>M \rho\left(2^{-j}\right)\right),
$$

this convergence follows easily from the dominated convergence theorem provided that the right hand side of (3.2) be finite for some $M=M_{0}>0$. Recalling that Card $V_{j} \sim\left(1-2^{-d}\right) 2^{j d}$ and using (3.1), this last requirement is satisfied as soon as $R\left(2 M_{0}\right)$ is finite.

Invoking again Corollary 1 and continuity in probability, we see that $\xi$ has a version in $H_{\rho}^{o}(B)$ if and only if $\left\|\xi-E_{J} \xi\right\|_{\rho}^{\text {seq }}$ goes to zero almost surely. But this sequence being decreasing, this is equivalent to its convergence in probability. Clearly, this convergence holds if for every $\varepsilon>0$,

$$
\sum_{j=0}^{\infty} \sum_{v \in V_{j}} P\left(\left\|\lambda_{j, v}(\xi)\right\|_{B}>\varepsilon \rho\left(2^{-j}\right)\right)<\infty,
$$

which in turn follows from $R(2 \varepsilon)<\infty$.

When $\Psi$ is non-increasing and $\sigma$ non-decreasing, a comparison between series and integral using (3) allows us to replace $R(u)$ by $I(u)$ in the above estimates.

Remarks on the optimality. From the very elementary nature of the proof it is easy to understand why the results are optimal in some sense. Indeed the only gaps in the proof between sufficient and necessary conditions are in the use of (3.2) and (3.1). But (3.2) is nothing else than the majorization of the probability of a denumerable union by the sum of probabilities. So if we do not know anything on the dependence structure of these events (which is the case since assumptions on $\xi$ involve only its three dimensional distributions) this bound cannot be improved in general. Concerning (3.1), it is clear that for reasonable $\Psi$ we can always construct $\xi$ such that (3.1) becomes an equality when reduced to $t \in \cup_{j} V_{j}, t \pm h=t^{ \pm}$.

Example. Let $\{\xi(t), t \in \mathbb{R}\}=\left\{\xi_{k}(t), t \in \mathbb{R}\right\}_{k=1}^{\infty}$ be a sequence of independent Gaussian stationary processes. For each $k \geq 1$, let $r_{k}$ denote the covariance function of $\left\{\xi_{k}(t), t \in \mathbb{R}\right\}$. Assume that $\sum_{k=1}^{\infty} \exp \left\{-\varepsilon / r_{k}(0)\right\}<\infty$ for each $\varepsilon>0$. Then for each $t \in \mathbb{R}$, the sequence $\xi(t)=\left\{\xi_{k}(t)\right\}_{k=1}^{\infty}$ is a.s. in $c_{0}$ (see Vakhania 
[15]). We consider the problem when for a fixed interval $[a, b]$ the process $\{\xi(t), t \in[a, b]\}$ has a version satisfying certain Hölder condition. Set

$$
\sigma_{k}^{2}(h)=2\left(r_{k}(2 h)-4 r_{k}(h)+3 r_{k}(0)\right) .
$$

Assume that for each $\varepsilon>0$

$$
\sum_{k=1}^{\infty} \sum_{j=1}^{\infty} 2^{j} \sigma_{k}\left(2^{-j}\right) \exp \left\{-\varepsilon \rho^{2}\left(2^{-j}\right) / \sigma_{k}^{2}\left(2^{-j}\right)\right\}<\infty .
$$

Then by Theorem 1 the process $\{\xi(t), 0 \leq t \leq 1\}$ admits a version in $H_{\rho}^{o}\left([0,1] ; c_{0}\right)$.

To prove this claim, we shall check the conditions of Theorem 1. For all $r>0, t \in[0,1]$ and $h, 0 \leq h \leq 1-t$ we have

$$
P\left(\left\|\Delta_{h}^{2} \xi(t)\right\|_{c_{0}}>r \sigma(h)\right) \leq \sum_{k=1}^{\infty} P\left(\left|\Delta_{h}^{2} \xi_{k}(t)\right|>r \sigma(h)\right) .
$$

Since $\Delta_{h}^{2} \xi_{k}(t)$ has a normal distribution with mean zero and variance $\sigma_{k}^{2}(h)$, we have

$$
P\left(\left|\Delta_{h}^{2} \xi_{k}(t)\right|>\lambda\right) \leq \frac{2}{\sqrt{2 \pi}} \frac{\sigma_{k}(h)}{\lambda} \exp \left\{-\lambda^{2} / 2 \sigma_{k}^{2}\right\}, \quad \lambda>0 .
$$

Now the conditions of Theorem 1 are satisfied by (3.3), (3.4) and (3.5), so the result follows.

As a special case, assume that for each $k \geq 1$ the process $\left\{\xi_{k}(t), t \in \mathbb{R}\right\}$ is the Ornstein-Uhlenbeck process defined as a stationary solution of the stochastic differential equation

$$
d \xi_{k}(t)=-\lambda_{k} \xi_{k}(t) d t+\left(2 \gamma_{k}\right)^{1 / 2} d W_{k}(t),
$$

where $\left\{W_{k}(t), t \in \mathbb{R}\right\}_{k=1}^{\infty}$ are independent Wiener processes. In this case we have $r_{k}(h)=\left(\gamma_{k} / \lambda_{k}\right) \exp \left\{-\lambda_{k}|h|\right\}$. Hence, if for each $\varepsilon>0$

$$
\sum_{k} \exp \left\{-\varepsilon / \gamma_{k}\right\}<\infty
$$

then the process $\{\xi(t), t \in[a, b]\}$ has a version in $H_{\rho}^{o}\left([a, b] ; c_{0}\right)$, with $\rho(h)=$ $\left.(h|\log h|)^{1 / 2}\right)$. It is easy to consider some weaker conditions like for each $\varepsilon>0$

$$
\sum_{k} \exp \left\{-\varepsilon \lambda^{1-\alpha} / \gamma_{k}\right\}<\infty,
$$

where $0<\alpha \leq 1$, which yields a weaker Hölder regularity of the OrnsteinUhlenbeck process $\{\xi(t), t \in \mathbb{R}\}$.

The next theorem is a straightforward application of Theorem 1 . When $B=\mathbb{R}$, it allows a comparison with classical Kolmogorov's and Ibragimov's results. 
Theorem 2. Let $\xi=\{\xi(t), t \in T\}$ be a B-valued random field, continuous in probability. Assume there exist a function $\sigma:[0,1] \rightarrow \mathbb{R}^{+}, \sigma(0)=0$ and a constant $p>0$ such that for all $r>0, t \in T, h \in C_{t}$,

$$
P\left(\left\|\Delta_{h}^{2} \xi(t)\right\|_{B}>r\right) \leq \frac{\sigma^{p}(|h|)}{r^{p}} .
$$

Suppose that

$$
R(1):=\sum_{j=0}^{\infty} \frac{2^{j d} \sigma^{p}\left(2^{-j}\right)}{\rho^{p}\left(2^{-j}\right)}<\infty .
$$

Then $\xi$ has a version $\tilde{\xi}$ in $H_{\rho}^{o}(B)$. Moreover, there is a constant $C$ such that $P\left(\|\tilde{\xi}\|_{\rho}>u\right) \leq C u^{-p}$ for each $u>0$.

Proof. Applying Theorem 1 with $\Psi(r)=r^{-p}$, the functional $R(u)$ is written here

$$
R(u):=\sum_{j=0}^{\infty} \frac{2^{j d} \sigma^{p}\left(2^{-j}\right)}{\rho^{p}\left(2^{-j}\right)} u^{-p}
$$

whose finiteness does not depend on the value of $u>0$. This explains why (3.6) cannot provide a version $\tilde{\xi}$ in $H_{\rho}(B)$ for some $\rho$ such that $\tilde{\xi}$ does not belong to the corresponding $H_{\rho}^{o}(B)$.

The tail behavior of $\|\tilde{\xi}\|_{\rho}$ results clearly from the same behavior of $\|\xi\|_{\rho}^{\text {seq }}$ which, in turn, follows from

$$
P\left(\|\xi\|_{\rho}^{\text {seq }}>u\right) \leq R(2 u)=2^{-p} R(1) u^{-p} .
$$

Remark. If (3.6) is replaced by the stronger assumption

$$
\mathbf{E}\left\|\Delta_{h}^{2} \xi(t)\right\|_{B}^{p}<\sigma^{p}(|h|),
$$

then a straightforward estimate of $\mathbf{E}\left(\|\xi\|_{\rho}^{\text {seq }}\right)^{p}$ gives $\mathbf{E}\|\tilde{\xi}\|_{\rho}^{p}<\infty$.

Corollary 2. Suppose $\xi$ satisfies (3.6) with $\sigma^{p}(|h|)=C|h|^{d+\delta}$ for some positive constants $C>0,0<\delta<p$. Then $\xi$ has a version $\tilde{\xi}$ in the Hölder space $H_{\rho}^{o}\left([0,1]^{d} ; B\right)$, where $\rho(|h|)=|h|^{\delta / p} \ln ^{\beta}(a /|h|)$ and $\beta>1 / p$.

A comparison with Corollary 2 in the paper [6] by Ibragimov is natural here. He obtained (in the case $B=\mathbb{R}$ ) the existence of a version in $H_{\rho}(\mathbb{R})$ under (3.7) with $p>1$ and the integral condition

$$
\int_{0}^{1} \frac{\sigma(u)}{\rho(u) u^{1+d / p}} d u<\infty .
$$

For the scale of moduli $\rho(|h|)=|h|^{\alpha} \ln ^{\beta}(a /|h|)$ and $\sigma^{p}(|h|)=C|h|^{d+\delta}$ this provides a version of $\xi$ in $H_{\rho}^{o}(\mathbb{R})$, where $\left.\rho(h)=h^{\delta / p} \ln ^{\beta}(a / h)\right)$ and $\beta>1$. 
Example. Let $\{\xi(t), t \in \mathbb{R}\}=\left\{\xi_{k}(t), t \in \mathbb{R}\right\}_{k=1}^{\infty}$ be a sequence of independent symmetric $p$-stable stationary processes. Let, for each $k \geq 1$, the process $\left\{\xi_{k}(t), t \in \mathbb{R}\right\}$ has the stochastic representation

$$
\left\{\xi_{k}(t), t \in \mathbb{R}\right\}=\left\{\int_{E} f_{k}(t, u) M_{k}(d u), t \in \mathbb{R}\right\},
$$

where $(E, \mathcal{E})$ is a measurable space, $M_{k}$ is a symmetric $p$-stable random measure with $\sigma$-finite spectral measure $m_{k}$. If

$$
\sum_{k=1}^{\infty} \int_{E}\left|f_{k}(t, u)\right|^{p} m_{k}(d u)<\infty
$$

then $\left(\xi_{k}(t), k \geq 1\right)$ is a.s. in $\ell_{r}$ for each $r>p$. Set

$$
\sigma_{k}^{p}(t, h)=\int_{E}\left|f_{k}(t+h, x)+f_{k}(t-h, x)-2 f_{k}(t, x)\right|^{p} m_{k}(d x)
$$

and assume that there exists a function $\sigma$ such that

$$
\sup _{0 \leq t \leq 1} \sum_{k=1}^{\infty} \sigma_{k}^{p}(t, h) \leq \sigma(h) \text { and } \sum_{j=0}^{\infty} 2^{j} \frac{\sigma^{p}\left(2^{-j}\right)}{\rho^{p}\left(2^{-j}\right)}<\infty .
$$

Then the process $\{\xi(t), t \in[0,1]\}$ has a version in $H_{\rho}^{o}\left([0,1] ; \ell_{r}\right)$ for $r>p$. Indeed, since $\left\{\Delta_{k}^{2} \xi_{k}(t)\right\}_{k=1}^{\infty}$ is a stable random element in $\ell_{r}$ with exponent $p$ and spectral measure $m=\sum_{k=1}^{\infty} \delta_{e_{k} \sigma_{k}}$, where $\left(e_{k}\right)$ is the coordinate basis in $\ell_{r}$, we have (see, e.g., Samorodnitsky and Taqqu [13])

$$
P\left(\left\|\Delta_{h}^{2} \xi(t)\right\|_{\ell_{r}}>\lambda\right) \leq c \lambda^{p} \int_{\ell_{r}}\|x\|_{\ell_{r}}^{p} m(d x)=C \lambda^{p} \sum_{k=1}^{\infty} \sigma_{k}^{p}(h) .
$$

Hence the result follows by Theorem 1.

Recall that a Young function $\phi$ is a convex increasing function on $\mathbb{R}^{+}$such that $\phi(0)=0$ and $\lim _{t \rightarrow \infty} \phi(t)=\infty$. If $Z$ is a real valued random variable such that $E \phi(|Z| / c)<\infty$ for some $c>0$, then its $\phi$-Orlicz norm is $\|Z\|_{\phi}:=\inf \{c>$ $0: \mathbf{E} \phi(|Z| / c) \leq 1\}$. When $Z$ is $B$-valued, its $\phi$-Orlicz norm is defined similarly, replacing $|Z|$ by $\|Z\|_{B}$. Using the definition of the Orlicz norm, the continuity of $\phi$ and Beppo Levi's theorem, it is easy to see that $\mathbf{E} \phi\left(\|Z\|_{B} /\|Z\|_{\phi}\right) \leq 1$, from which we get $P\left(\|Z\|_{B}>r\right) \leq\left\{\phi\left(r /\|Z\|_{\phi}\right)\right\}^{-1}$.

Theorem 3. Let $\phi$ be a Young function. Assume that the B-valued random field $\xi=\left(\xi_{t}, t \in T\right)$ is continuous in probability and satisfies the condition: for each $t \in T$ and $h \in C_{t}$,

$$
\left\|\Delta_{h}^{2} \xi(t)\right\|_{\phi} \leq \sigma(|h|)
$$

If for some $u>0$,

$$
\sum_{j=0}^{\infty} \frac{2^{j d}}{\phi\left(u \frac{\rho}{\sigma}\left(2^{-j}\right)\right)}<\infty
$$


then $\xi$ admits a version with almost all paths in $H_{\rho}(B)$. If (3.9) holds for each $u>0$, then $\xi$ has a version in $H_{\rho}^{o}(B)$.

Proof. Under (3.8), Theorem 1 applies with $\Psi(r)=1 / \phi(r)$.

Of course, the scope of Theorem 3 covers the case of strong $p$-moments $(p>2)$ with (3.8) replaced by (3.7). But its main practical interest is in the case of exponential Orlicz norms as in Corollaries 3 and 4 below. Then the convergence of series (3.9) can be checked through the following routine test.

Lemma 3. Let $\Psi$ be of the form $\Psi(r)=c \exp \left(-b r^{\gamma}\right)$ for some positive constants $b, c, \gamma$. Consider the series

$$
R(u)=\sum_{j=0}^{\infty} 2^{j d} \Psi\left(u \frac{\rho}{\sigma}\left(2^{-j}\right)\right)
$$

and put

$$
L(j):=\frac{\rho\left(2^{-j}\right)}{j^{1 / \gamma} \sigma\left(2^{-j}\right)} .
$$

(i) $R(u)$ converges for some $0<u_{0}<\infty$ if and only if $\liminf _{j \rightarrow \infty} L(j)>0$;

(ii) $R(u)$ converges for every $0<u<\infty$ if and only if $\lim _{j \rightarrow \infty} L(j)=\infty$.

The proof is elementary and shall be omitted. Observe that the qualitative form of the result does not depend on $d$. This parameter is involved only in the explicit determination of $u_{0}$.

Corollary 3. If the B-valued random field $\xi$ is continuous in probability and satisfies (3.8) for the Young function $\phi_{\gamma}(r)=\exp \left(r^{\gamma}\right)-1(\gamma>0)$ and $\sigma(|h|)=|h|^{\alpha}(0<\alpha<1)$, then $\xi$ has a version in $H_{\rho}^{o}(T ; B)$, where $\rho(h)=$ $\left.|h|^{\alpha} \ln ^{1 / \gamma}(a /|h|)\right)$.

This improves our previous result [11, Th. 11].

The case of Gaussian random fields is of special interest.

Corollary 4. Assume that the Gaussian B-valued random field $\xi=\left(\xi_{t}, t \in\right.$ $T)$ is continuous in probability and satisfies the condition: for each $t \in T$ and $h \in C_{t}$,

$$
\mathbf{E}\left\|\Delta_{h}^{2} \xi(t)\right\|_{B}^{2} \leq \sigma^{2}(|h|) .
$$

(i) If $\liminf _{j \rightarrow \infty} \frac{\rho\left(2^{-j}\right)}{j^{1 / 2} \sigma\left(2^{-j}\right)}>0$, then $\xi$ admits a version in $H_{\rho}(B)$.

(ii) If $\lim _{j \rightarrow \infty} \frac{\rho\left(2^{-j}\right)}{j^{1 / 2} \sigma\left(2^{-j}\right)}=\infty$, then $\xi$ has a version in $H_{\rho}^{o}(B)$.

If for some $u>0$,

$$
\sum_{j=0}^{\infty} 2^{j d} \exp \left(-u^{2} \frac{\rho^{2}}{\sigma^{2}}\left(2^{-j}\right)\right)<\infty
$$


then $\xi$ admits a version in $H_{\rho}(B)$. If (3.11) holds for each $u>0$, then $\xi$ has a version in $H_{\rho}^{o}(B)$.

Proof. By (3.10) and the classical estimate of the tail of Gaussian random element in the Banach space $B$ (see, e.g., inequality (3.5) p. 59 in [8]),

$$
P\left(\left\|\Delta_{h}^{2} \xi(t)\right\|_{B}>r\right) \leq 4 \exp \left(-\frac{r^{2}}{8 \sigma^{2}(|h|)}\right),
$$

from which the result follows.

Example. Let $B$ be a separable Banach space and $Y$ a centered Gaussian random element in $B$ with distribution $\mu$. A $B$-valued Brownian motion with parameter $\mu$ is a Gaussian process $\xi$ indexed by $[0,1]$, with independent increments such that $\xi(t)-\xi(s)$ has the same distribution as $|t-s|^{1 / 2} Y$. Hence (3.10) holds with $\sigma(h)=h^{1 / 2} \mathbf{E}^{1 / 2}\|Y\|_{B}^{2}(h \geq 0)$. Choosing the modulus of smoothness $\rho(h)=\sqrt{h \ln (e / h)}$, we see that

$$
\lim _{j \rightarrow \infty} \frac{\rho\left(2^{-j}\right)}{j^{1 / 2} \sigma\left(2^{-j}\right)}=\frac{1}{\mathbf{E}^{1 / 2}\|Y\|_{B}^{2}}>0 .
$$

Hence by Corollary 4 (ii), the $B$ valued Brownian motion $\xi$ has a version in $H_{\rho}([0,1] ; B)$, where $\rho(h)=\sqrt{h \ln (e / h)}, h>0$. This result cannot be improved because of Lévy's theorem on the modulus of uniform continuity of the standard Brownian motion.

Remark. One may ask what happens with Problem (I) when $\rho$ is a general modulus of smoothness, i.e., simply non-decreasing on $[0,1]$ and continuous at 0 . In this case we can always replace the inequality $\|x\|_{\rho} \leq b\|x\|_{\rho}^{\text {seq }}$ of Proposition 2 by

$$
\|x\|_{\rho} \leq 3 \sum_{j=0}^{\infty} \frac{1}{\rho\left(2^{-j}\right)} \max _{v \in V_{j}}\left\|\lambda_{j, v}(x)\right\|_{B}=:\|x\|_{\rho}^{\ell_{1}} .
$$

This approach was adopted in the pioneering paper by Delporte [4] in the case $d=1, B=\mathbb{R}$. Because the norm $\|x\|_{\rho}^{\ell_{1}}$ is clearly weaker than $\|x\|_{\rho}^{\text {seq }}$, the price paid for this greater generality is the loss of precision in the topology. For instance, in the situation of Corollary 2, this gives the same results as in [6].

\section{ACKNOWLEDGEMENT}

This research was supported by the cooperation agreement CNRS/LITHUANIA (4714).

\section{REFERENCES}

1. R. Bonic, J. Frampton, and A. Tromba, $\Lambda$-manifolds. J. Functional Analysis 3(1969), 310-320.

2. Z. Ciesielski, On the isomorphisms of the spaces $H_{\alpha}$ and m. Bull. Acad. Pol. Sci. Ser. Sci. Math. Phys. 8(1960), 217-222. 
3. Z. Ciesielski, Hölder conditions for realizations of Gaussian processes. Trans. Amer. Math. Soc. 99(1961), 403-413.

4. J. Delporte, Fonctions aléatoires presque sûrement continues sur un intervalle fermé. Ann. Inst. H. Poincaré Sect. B 1(1964), 111-215.

5. R. V. ERICKSON, Lipschitz smoothness and convergence with applications to the central limit theorem for summation processes. Ann. Probab. 9(1981), 831-851.

6. I. Ibragimov, On smoothness conditions for trajectories of random functions. Theor. Probab. Appl. 28(1984), 240-262.

7. G. Kerkyacharian and B. Roynette, Une démonstration simple des théorèmes de Kolmogorov, Donsker et Ito-Nisio. C. R. Acad. Sci. Paris Série I 312(1991), 877-882.

8. M. Ledoux and M. Talagrand, Probability in Banach spaces. Springer-Verlag, Berlin, Heidelberg, 1991.

9. Pн. NobÉLIs, Fonctions aléatoires lipschitziennes. Lecture Notes in Math. 850, 38-43, Springer Verlag, Berlin, Heidelberg, New York, 1981.

10. A. RaČKauskas and Ch. Suquet, Central limit theorem in Hölder spaces. Probab. Math. Statist. 19(1999), No. 1, 133-152.

11. A. RaČKauskas and Ch. Suquet, Random fields and central limit theorem in some generalized Hölder spaces. B. Grigelionis et al. (Eds.), Prob. Theory and Math. Statist., Proceedings of the 7th Vilnius Conference (1998), 599-616, TEV, Vilnius and VSP, Utrecht, 1999.

12. A. RaČKauskas and $\mathrm{CH}$. Suquet, On the Hölderian functional central limit theorem for Banach space valued i.i.d. random variables. Pub. IRMA Lille 50-III, to appear in Proceedings of the Fourth Hungarian Colloquium on Limit Theorems of Probability and Statistics, 1999.

13. G. SAmorodnitsky and M. S. TAqQU, Stable non-Gaussian random processes. Stochastic models with infinite variance, Chapman \& Hall, 1994.

14. Z. Semadeni, Schauder bases in Banach spaces of continuous functions. Lecture Notes in Math. 918, Springer Verlag, Berlin, Heidelberg, New York, 1982.

15. N. N. VAKhania, Probability distributions on linear spaces. North-Holland, New York/Oxford, 1981; Russian original: Metsniereba, Tbilisi, 1971.

(Received 22.02.2001)

Authors' addresses:

Alfredas Račkauskas

Vilnius University

Naugarduko 24, LT-2006, Vilnius, Lithuania

Institute of Mathematics and Informatics

Akademijos 4, LT-2600, Vilnius, Lithuania

E-mail: alfredas.rackauskas@maf.vu.lt

Charles Suquet

Université des Sciences et Technologies de Lille

Statistique et Probabilités E.P. CNRS 1765

Bât. M2, U.F.R. de Mathématiques

F-59655 Villeneuve d'Ascq Cedex, France

E-mail: Charles.Suquet@univ-lille1.fr 